\title{
The effect of subcutaneous fat on electrical impedance myography when using a handheld electrode array: The case for measuring reactance
}

\section{Citation}

Sung, Minhee, Andrew J. Spieker, Pushpa Narayanaswami, and Seward B. Rutkove. 2013.

"The Effect of Subcutaneous Fat on Electrical Impedance Myography When Using a Handheld Electrode Array: The Case for Measuring Reactance." Clinical Neurophysiology 124 (2) (February): 400-404. doi:10.1016/j.clinph.2012.07.013.

\section{Published Version}

doi:10.1016/j.clinph.2012.07.013

\section{Permanent link}

http://nrs.harvard.edu/urn-3:HUL.InstRepos:34216311

\section{Terms of Use}

This article was downloaded from Harvard University's DASH repository, and is made available under the terms and conditions applicable to Other Posted Material, as set forth at http:// nrs.harvard.edu/urn-3:HUL.InstRepos:dash.current.terms-of-use\#LAA

\section{Share Your Story}

The Harvard community has made this article openly available.

Please share how this access benefits you. Submit a story.

Accessibility 


\title{
The effect of subcutaneous fat on electrical impedance myography when using a handheld electrode array: the case for measuring reactance
}

\author{
Minhee Sung, BS, Andrew J. Spieker, BS, Pushpa Narayanaswami, MD, and Seward B. \\ Rutkove, MD \\ Department of Neurology, Beth Israel Deaconess Medical Center, Boston, MA 02215
}

\begin{abstract}
Objective-Recent developments in electrical impedance myography (EIM) have led to the use of handheld electrode arrays (HEA) for data acquisition. Although preferable for several reasons, this approach tends to be more affected by subcutaneous fat (SF) than the original approach in which the impedance-measuring electrodes are widely spaced. In this study, we seek to identify the EIM parameter least impacted by subcutaneous fat (SF) when using an HEA.
\end{abstract}

Methods-18 normal subjects underwent $50 \mathrm{kHz}$ EIM and ultrasound of the medial gastrocnemius muscles on the dominant side. Coefficients of determination $\left(R^{2}\right.$ values) were calculated for each of the three major EIM variables (reactance, resistance, and phase) and SF thickness.

Results-For both resistance and phase, a strong relationship to SF thickness was observed ( $R^{2}$ $=0.64$ and $R^{2}=0.70$, respectively, $\mathrm{p}<0.001$ for both). In contrast, for reactance, the relationship was non-significant, with $R^{2}=0.07, \mathrm{p}=0.30$.

Conclusions-Unlike resistance and phase, both of which are highly impacted by SF thickness, the reactance shows no significant relationship.

Significance-Future clinical studies employing HEA's to perform EIM should evaluate alterations in reactance over those in resistance and phase.

\section{Keywords}

electrical impedance; subcutaneous fat; handheld array

\section{INTRODUCTION}

Electrical impedance myography (EIM), a technique based on the application and measurement of high-frequency electrical current across discrete areas of muscle, is finding

\footnotetext{
(c) 2012 International Federation of Clinical Neurophysiology. Published by Elsevier Ireland Ltd. All rights reserved. Corresponding Author: Seward B. Rutkove, MD, Beth Israel Deaconess Medical Center, Department of Neurology, 330 Brookline Avenue, Boston, MA 02215, Telephone: 617-667-8130; Fax: 617-667-3175; srutkove@ bidmc.harvard.edu.

Conflicts of Interest: Dr. Rutkove has equity interest and provides consulting services to Convergence Medical Devices, Inc which develops impedance-measuring devices. However, all of the research completed in this study was performed with a device from another company (Impedimed, Inc) with which Dr. Rutkove has no affiliation.

Publisher's Disclaimer: This is a PDF file of an unedited manuscript that has been accepted for publication. As a service to our customers we are providing this early version of the manuscript. The manuscript will undergo copyediting, typesetting, and review of the resulting proof before it is published in its final citable form. Please note that during the production process errors may be discovered which could affect the content, and all legal disclaimers that apply to the journal pertain.
} 
wider application for the quantification and assessment of neuromuscular disease (Rutkove 2009). EIM appears well-suited for monitoring disease progression over time and as an outcome measure for assessing drug efficacy, as supported by studies in amyotrophic lateral sclerosis (ALS) (Rutkove et al, 2007) and spinal muscular atrophy (Rutkove et al, 2012). The technique also holds promise in the initial diagnosis of focal and generalized disorders (Garmirian et al, 2009).

EIM assesses the flow of current through localized areas of tissue, and like most impedancebased techniques, a four-electrode approach is used in which the outer two electrodes are current-emitting and the inner two are voltage-sensing (Lukaski 1999). In our original work developing EIM, we placed the current-electrodes at a distant site from the voltage measuring electrodes (for example, on the palm of each hand with the voltage electrodes placed over the biceps) (Rutkove et al, 2002; Esper et al, 2006). This had the advantages of ensuring that current was flowing through the highly conductive muscle at the point it reached the voltage electrodes and that the subcutaneous fat (SF) had minimal impact on any of the major EIM variables, the phase, resistance or reactance (see Figure 1A) (Tarulli et al, 2007). However, more recent studies have used a "near-current electrode montage" in which all four electrodes are placed in close proximity to one another (Rutkove et al, 2010). Such an approach is advantageous since it allows for improved localized muscle assessment and the measurement of muscle anisotropy by making it possible to apply the electrical current at specific angles relative to the major muscle fiber direction (Garmirian et al, 2009). It also reduces the impact of joint position or of metal in joint replacements, and minimizes inductive artifacts. However, the principal reason for using such a montage is that it allows for all four electrodes to be placed on a fixed handheld array (HEA), making measurements still simpler and faster to perform (Narayanaswami et al, 2012). The major drawback to this approach, however, is that SF may have a greater impact on the data since the electrical current will always take the path of least resistance; with the current electrodes relatively close to one another, there will be greater flow through the subcutaneous tissue (see Figure 1B) with the measured voltages representing a mix of data from muscle and fat.

Recognizing this potential limitation, we have been measuring SF thickness using ultrasound or skin calipers in all our work, and, where appropriate, including this as a covariate in our analyses (Rutkove et al, 2012). Although we have been generally using the phase value as the major EIM parameter in most analyses, for reasons discussed in greater depth below, we hypothesized that the reactance would be least affected by the presence of SF when using an HEA, thus making it the preferred parameter when performing EIM with a handheld device.

\section{MATERIALS AND METHODS}

\section{Approvals}

The study was approved by the institutional review board of Beth Israel Deaconess Medical Center (BIDMC), and written informed consent was obtained from all participants.

\section{Subjects}

Healthy volunteers aged 18-85 without a past history of radiating lower back pain or of a previously diagnosed neuromuscular disease were recruited through advertisement or through the EMG laboratory if they had normal studies and had been referred for a disorder not anticipated to involve the gastrocnemius muscle. The gastrocnemius muscle was specifically chosen to study since EIM measurements are straightforward to perform on this muscle, and the SF thickness overlying the muscle varies considerably across the population. After signing an informed consent form, subjects underwent a brief review of 
their medical and neurological history. A neurological examination was then performed, including evaluation of cranial nerves, strength, reflexes and sensation of both the upper and lower extremities; gait was also assessed.

For consistency, the gastrocnemius on the dominant side (as defined by upper extremity handedness) was studied in all subjects; in subjects in whom upper extremity handedness was uncertain, the right side was measured.

\section{EIM Measurements}

EIM measurements were obtained using the Imp SFB7@ bioimpedance spectroscopy device (Impedimed, San Diego, CA). This device measures resistance $(R)$ and reactance $(X)$ at multiple frequencies ranging from $3 \mathrm{kHz}$ to $1 \mathrm{MHz}$, from which phase $(\theta)$ is calculated $(\theta=$ $\arctan (X / R))$. The measurements were made with a handheld electrode array (HEA) as previously described (Narayanaswami et al, 2012). The HEA consists of four stainless steel electrodes, the inner two serving as voltage-measuring electrodes and the outer two serving as current-emitting electrodes (see Figure 2A). The electrodes were $0.75 \mathrm{~cm}$ wide by $2.5 \mathrm{~cm}$ long and were arranged such that the inner electrodes are $3 \mathrm{~cm}$ apart and the outer electrodes are $1.5 \mathrm{~cm}$ peripheral to that. Receptacles at the top of the handle received the plugs from the Imp SFB7 ${ }^{B}$ device (see Figure 2B). The HEA itself had a negligible $(<1 \mathrm{ohm})$ internal impedance at $50 \mathrm{kHz}$. Prior to applying the array, the skin of overlying the gastrocnemius was moistened with isotonic saline to ensure good electrical contact.

\section{Skin-Subcutaneous Fat Layer Thickness Measurement}

SF thickness was measured in the same region in which the EIM data was collected via ultrasound with the Terason 2000 Handheld Ultrasound System (Terason Ultrasound, Teratech, Burlington, MA). The 5-MHz probe was placed at the approximate midpoint of the bulk of the relaxed muscle, perpendicularly to the direction of the muscle fibers. The subject was asked to briefly contract and relax his or her muscle to assist with the identification of the SF-muscle interface. Once a quality ultrasound picture was captured, electronic calipers from the Terason software were used to measure the SF thickness.

\section{Data Analysis}

Data obtained at $50 \mathrm{kHz}$ frequency was used in these analyses. Pearson correlation was used to determine the coefficient of determination between all EIM parameters and SF thickness. For all statistical tests, significance was accepted at $\mathrm{p}<0.05$, two-tailed. Analyses were performed using MATLAB (Mathworks, Inc., Natick, MA).

\section{RESULTS}

\section{Subject Demographics}

18 healthy adult volunteers were studied ( 9 male and 9 female). Subjects aged from 19 to 83 years with a mean age of 50.7 years.

\section{Subcutaneous fat thickness}

We first confirmed that there was a normal distribution of fat amongst the healthy volunteers. This data is shown in Figure 3A as a histogram. As can be seen, there is indeed a wide spread in the data, with fat thickness ranging from $2 \mathrm{~mm}$ to $12 \mathrm{~mm}$ with a mean of 6.28 $\mathrm{mm}$. Figure 3B shows an example of SF measured with ultrasound using the electronic calipers. 


\section{Correlation between subcutaneous fat thickness and EIM data at $50 \mathrm{kHz}$}

Figure 4 shows the correlation between SF thickness and the EIM values for the 18 gastrocnemius muscles evaluated. There is a very strong relationship between the resistance/ phase at $50 \mathrm{kHz}$ and the SF thickness, with coefficient of determination $\left(R^{2}\right)$ values of 0.64 and 0.70 respectively ( $\mathrm{p}<0.001$ for both). However, for reactance, the $R^{2}$ value is only $0.07, \mathrm{p}=0.30$. The relationship between $\mathrm{SF}$ thickness and age was also evaluated since increasing age can be associated with muscle atrophy and increasing SF thickness. However, there was only a very weak, non-significant relationship between SF thickness and age in this group $\left(R^{2}=0.054, \mathrm{p}=0.35\right)$. Age correlated only weakly to resistance $\left(R^{2}=0.24, \mathrm{p}=\right.$ $0.04)$, but not significantly to phase $\left(R^{2}=0.06, \mathrm{p}=0.33\right)$ or to reactance $\left(R^{2}=0.17, \mathrm{p}=\right.$ $0.09)$.

\section{DISCUSSION}

These data support the concept that of the 3 major $50 \mathrm{kHz}$ EIM parameters obtained, the reactance data is the least impacted by SF thickness when using a handheld array. On the other hand, both the resistance and the phase show relatively strong associations with SF. In the case of phase, for example, about $70 \%$ of its variation amongst healthy individuals can be explained by the SF thickness alone.

These relationships assume that there is no association between muscle health and the amount of SF thickness amongst these healthy volunteers. While that is likely to be true for the most part, variations in the state and composition of the muscle even amongst these normal individuals can be expected to affect the EIM measurements to some extent. For example, it is possible that those individuals with greater SF thickness also led more sedentary lifestyles and did not exercise regularly. Whereas this possibility could confound our analyses, the fact that the reactance appears to be relatively insensitive to SF thickness but has already been shown to be sensitive to muscle condition in other work (Wang et al, 2011), suggests that is likely not playing a major role.

The explanation for these differences among the three EIM variables lies in the inherent passive electrical properties, the conductivity and permittivity, of the muscle and fat. The conductivity represents the inherent ability of a tissue to conduct an electrical current at a given frequency and mostly impacts the measured resistance, being related to the reciprocal of that value (as conductivity increases, the measured resistance decreases). The permittivity is a measure of that tissue's polarizability, similar in some respects to capacitance, and mainly impacts the measured reactance. These two values interact in complex ways depending on the frequency of electrical current applied. Fat and muscle permittivity and conductivity spectra are quite different, and thus at $50 \mathrm{kHz}$, the characteristics of the tissue balance in such a way as to make the reactance only minimally affected by SF thickness.

Much of our focus in past work has been on the phase rather than the reactance. We chose to focus on phase because of our use of "far" current electrodes (Figure 1A) and the fact that in such a set-up the SF does not impact the data in a substantial way (Tarulli et al, 2007). Secondly, the phase has the added benefit of potentially cancelling out simple volumetric effects (Shiffman et al, 1999) since such variations would be expected to alter the resistance and reactance similarly. For example, decreasing muscle size alone would be anticipated to increase the measured resistance and the measured reactance, the effect of which would be at least partially cancelled out in the calculation of the phase. Third, many of our studies have been longitudinal in nature, following individual patients over time, and in which only SF thickness would be expected to remain stable. Finally, and perhaps most importantly, the phase has borne itself out as a clinically meaningful parameter with its correlating strongly to clinical status in a variety of diseases. 
Unlike studies in humans, in animal studies, by necessity, we have utilized four electrodes in close proximity to one another, similar to an HEA. In these studies, reactance like phase, is also sensitive to disease status. For example, in ALS rats, along with reductions in phase with progressive disease, we also identified significant reductions in reactance (Wang et al, 2011). Although specific values were not reported previously at $50 \mathrm{kHz}$, in reanalyzing data acquired in that study, we have found that $50 \mathrm{kHz}$ reactance decreases on average $0.27 \%$ / day; reactance also correlates strongly to MUNE (with a Spearman rank correlation of rho = 0.81). Importantly, the reactance values also do appear sensitive to disease status in human subjects (Garmirian et al, 2009; Rutkove et al, 2010). Regardless, it still remains to be determined whether or not reactance can serve as an effective variable for human disease assessment, and prospective studies utilizing this EIM parameter are needed.

As Figure 4 demonstrates, there is a considerable range of values for all 3 EIM parameters. While clearly the SF thickness contributes to this variability for resistance and phase, this is not the case for reactance. Importantly, a similar wide range of normal $50 \mathrm{kHz}$ phase values was also identified when performing EIM using our original approach (Rutkove et al, 2008) with the electrodes placed on the palms of the hands or the dorsum of the feet, in which SF thickness also contributed minimally to the range of acquired data (Tarulli et al, 2007). In both cases, the absence of such a clear relationship to SF thickness suggests that this variation is related to intrinsic properties of the muscle itself.

This study has several limitations. First, the reactance is smaller in magnitude than the resistance, thus potentially making it a noisier measure; this could have contributed to some of the lack of relationship to SF thickness. However, this effect is likely minor since the repeatability of the HEA on gastrocnemius is actually similar for both reactance and for phase (+/-9.8\% and +/-9.6, respectively, with intraclass correlation coefficients at or greater than 0.95 for both) (Narayanaswami et al, 2012). Second, measuring the SF itself involves some degree of error both in placing the transducer consistently each time and identifying exactly where the fat ends and the muscle fascia begins. However, this error should affect all EIM parameters, not just reactance. Third, we have reported data obtained at only a single frequency here $(50 \mathrm{kHz})$. This frequency is not necessarily the optimal frequency, but was chosen simply because single frequency impedance devices usually measure this one frequency and because muscle is most reactive at or near this frequency. Finally, in this study, we report data only on a single muscle, the gastrocnemius. We chose to study and analyze the data on this muscle specifically because amongst a normal population, gastrocnemius SF measurements have a fairly large range and are relatively normally distributed. Having a wide range of SF thickness allowed us to more effectively test our hypothesis. Preliminary analyses looking at data from other muscles showed similar results, however. Of note, the dominant side was chosen simply to help reduce any additional unforeseen confounders (e.g., differences in muscle condition).

As development of EIM proceeds, further analysis of the relationship between the various single and multiple frequency EIM parameters and the SF thickness will be needed. In addition, it will need to be determined whether the reactance, instead of the phase, can serve as the primary EIM outcome measure in human clinical studies. Nonetheless, from this work, it is now clear that given the $50 \mathrm{kHz}$ reactance's relative insusceptibility to SF thickness variations, it is a parameter that may be especially useful when performing EIM measurements using an HEA.

\section{Acknowledgments}

This study was supported by: Grant K24NS060951 and Grant R01NS055099, from the National Institutes of Health and the National Institute of Neurological Disorders and Stroke. 


\section{References}

Esper GJ, Shiffman CA, Aaron R, Lee KS, Rutkove SB. Assessing neuromuscular disease with multifrequency electrical impedance myography. Muscle Nerve. 2006; 34:595-602. [PubMed: 16881067]

Garmirian LP, Chin AB, Rutkove SB. Discriminating neurogenic from myopathic disease via measurement of muscle anisotropy. Muscle Nerve. 2009; 39:16-24. [PubMed: 19058193]

Lukaski HC. Requirements for clinical use of bioelectrical impedance analysis (BIA). Ann N Y Acad Sci. 1999; 873:72-76. [PubMed: 10372152]

Narayanaswami P, Spieker AJ, Mongiovi P, Keel JC, Muzin SC, Rutkove SB. Utilizing a handheld electrode array for localized muscle impedance measurements. Muscle Nerve. 2012; 46:257-63. [PubMed: 22806375]

Rutkove SB. Electrical Impedance Myography: Background, Current State, and Future Directions. Muscle Nerve. 2009; 40:936-946. [PubMed: 19768754]

Rutkove SB, Aaron R, Shiffman CA. Localized bioimpedance analysis in the evaluation of neuromuscular disease. Muscle Nerve. 2002; 25:390-397. [PubMed: 11870716]

Rutkove SB, Fogerson PM, Garmirian LP, Tarulli AW. Reference values for 50-kHZ electrical impedance myography. Muscle Nerve. 2008; 38:1128-32. [PubMed: 18642375]

Rutkove SB, Gregas MC, Darras BT. Electrical impedance myography in spinal muscular atrophy: A longitudinal study. Muscle \& nerve. 2012; 45:642-647. [PubMed: 22499089]

Rutkove SB, Shefner JM, Gregas M, Butler H, Caracciolo J, Lin C, et al. Characterizing spinal muscular atrophy with electrical impedance myography. Muscle Nerve. 2010; 42:915-921. [PubMed: 21104866]

Rutkove SB, Zhang H, Schoenfeld DA, Raynor EM, Shefner JM, Cudkowicz ME, et al. Electrical impedance myography to assess outcome in amyotrophic lateral sclerosis clinical trials. Clin Neurophysiol. 2007; 118:2413-2418. [PubMed: 17897874]

Shiffman C, Aaron R, Amoss V, Therrien J, Coomler K. Resistivity and phase in localized BIA. Phys Med Biol. 1999; 44:2409-2429. [PubMed: 10533919]

Tarulli AW, Chin AB, Lee KS, Rutkove SB. Impact of skin-subcutaneous fat layer thickness on electrical impedance myography measurements: An initial assessment. Clin Neurophysiol. 2007; 118:2393-2397. [PubMed: 17889597]

Wang LL, Spieker AJ, Li J, Rutkove SB. Electrical impedance myography for monitoring motor neuron loss in the SOD1 G93A amyotrophic lateral sclerosis rat. Clin Neurophysiol. 2011; 122:2505-2511. [PubMed: 21612980] 


\section{Highlights}

- Handheld electrode arrays can greatly speed electrical impedance measurements of muscles, but the standard parameter, phase, is very sensitive to subcutaneous fat thickness.

- Another electrical impedance parameter, the reactance, is relatively unaffected by subcutaneous fat thickness.

- The reactance appears to be the impedance parameter of choice when using a handheld electrode array. 


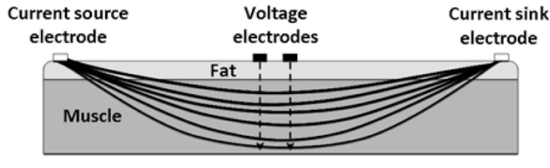

(A)

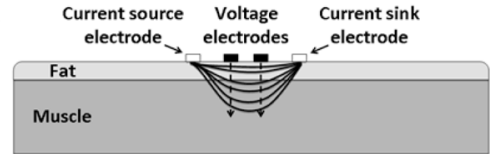

(B)

Figure 1.

(A) Current flow through tissue with current-emitting electrodes placed at a distance from the voltage measuring electrodes. Electrical current will seek out the path of least resistance; since muscle has much greater water content than fat, nearly all the current will pass through the muscle at the point where the voltage is being measured. (B) Current flow through tissue with current-emitting electrodes placed in close proximity to the voltage-measuring electrodes. With the electrodes much closer together, a considerable proportion of the applied current will be passing through the fat at the point where the voltage is being measured. 


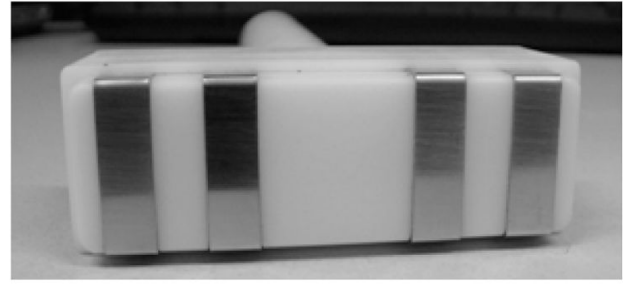

(A)

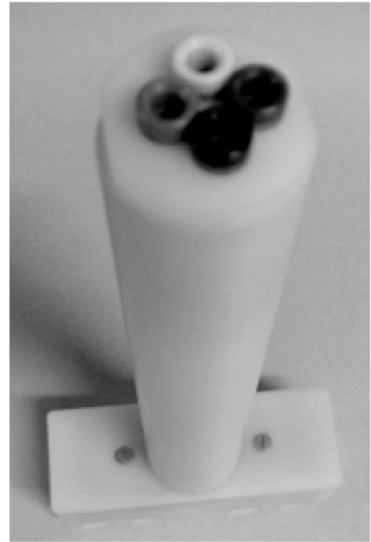

(B)

Figure 2.

A. Close up of the handheld electrode array designed for this study. The outer strips serve as the current-emitting electrodes and the inner as the voltage-measuring electrodes. B. Jacks at top of handle connect to cables from the impedance device, one jack for each electrode. 


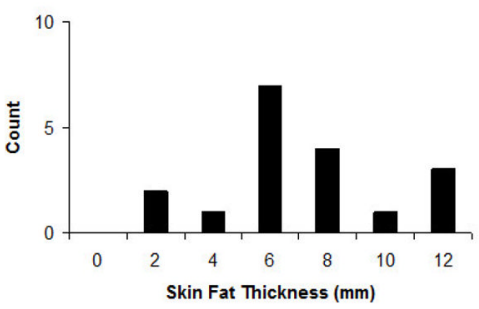

(A)

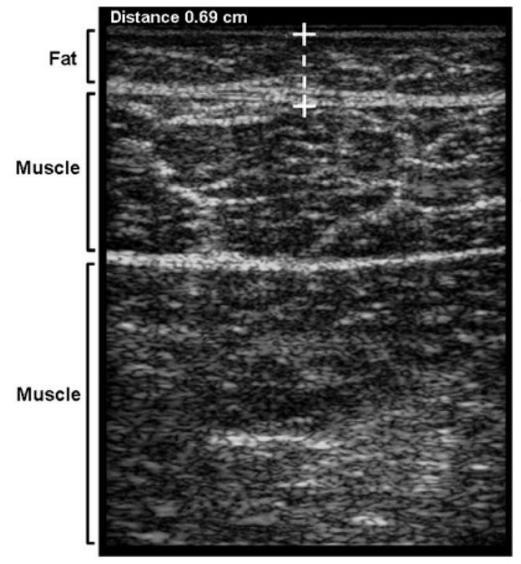

(B)

Figure 3.

(A) Histogram showing distribution of SF thickness overlying the gastrocnemius in the 18 normal subjects recruited. (B) Example of ultrasound data showing the measurement of the SF thickness in a 26-year-old woman. 

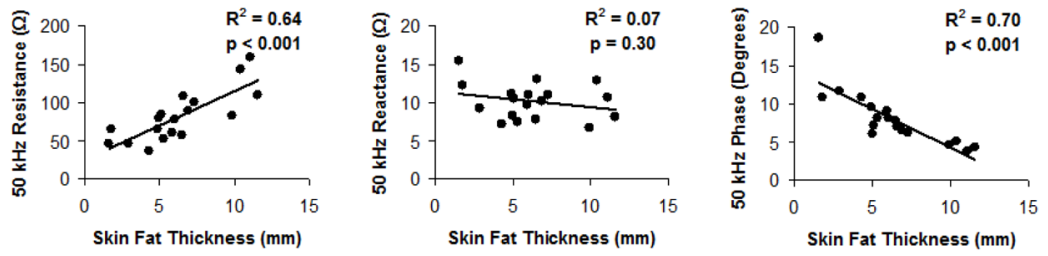

Figure 4.

Correlation plots for the three $50 \mathrm{kHz}$ EIM parameters versus SF thickness. Note the low value for reactance and relative high values for both phase and resistance. 